Revue québécoise de droit international

Quebec Journal of International Law

Revista quebequense de derecho internacional

\title{
L'Europe peut-elle faire l'économie du modèle fédéral? Conclusion de la table ronde des experts, avec quelques observations sur la gouvernance et la quête du changement
}

\author{
Mulry Mondélice
}

Numéro hors-série, novembre 2018

L'intégration européenne. Soixante ans du Traité de Rome : tous les chemins mènent-ils encore à Bruxelles?

URI : https://id.erudit.org/iderudit/1067276ar

DOI : https://doi.org/10.7202/1067276ar

Aller au sommaire du numéro

Éditeur(s)

Société québécoise de droit international

ISSN

0828-9999 (imprimé)

2561-6994 (numérique)

Découvrir la revue

Citer cet article

Mondélice, M. (2018). L'Europe peut-elle faire l'économie du modèle fédéral? Conclusion de la table ronde des experts, avec quelques observations sur la gouvernance et la quête du changement. Revue québécoise de droit international / Quebec Journal of International Law / Revista quebequense de derecho internacional, 357-368. https://doi.org/10.7202/1067276ar

\section{Résumé de l'article}

Cette contribution présente l'essentiel des réflexions interdisciplinaires présentées sur la question de savoir si l'Union peut faire l'économie du modèle fédéral lors de la table ronde des experts ayant réuni, le 28 avril 2017 à l’Université Laval, des panélistes de différents horizons lors du colloque international sur l'Union européenne - 60 ans du traité de Rome : "tous les chemins mènent-ils encore à Bruxelles?”. Un consensus s'est dégagé sur l'idée que l'Union ne peut pas faire l'économie du modèle fédéral. En témoignent des symptômes du fédéralisme : normes - même implicitement - , institutions, agences et politiques, faisant de l'UE une entité se rapprochant d'un État fédéral, avec une répartition des compétences entre l'Union et ses États membres. C'est le cas, par exemple, avec la place reconnue aux droits de la personne qu'illustrent, entre autres, l'existence de l'Agence des droits fondamentaux de l'Union européenne, mais également, l'action humanitaire avec l'Office d'aide humanitaire de l'Union, et la citoyenneté européenne et la mobilité des personnes, ce dernier domaine influençant dans une certaine mesure le Canada. Toutefois, ces symptômes du fédéralisme ne doivent pas faire oublier des pratiques, normes et politiques de l'Union, illustrant un certain fédéralisme en construction. En effet, les États membres ne se montrent pas tout à fait prêts à laisser trop de marge de manoeuvre à l'Union dans certaines de leurs compétences, notamment en matière d'affaires étrangères, ou dans des domaines sensibles comme l'immigration. Dès lors, cette situation plaçant l'Union dans un entre-deux n'est pas sans conséquences sur le poids de l'Union comme un acteur international. À une époque où le monde fait face à des crises complexes invitant à une gouvernance qui rassemble et mettant à l'épreuve les acteurs internationaux, il serait souhaitable que l'Union montre plus de leadership dans la quête du changement. Dans cette quête, le modèle de gouvernance de l'Union, entre les avancées et défis présents et à venir dans un monde complexe, demeure un enjeu majeur. 


\title{
L'EUROPE PEUT-ELLE FAIRE L'ÉCONOMIE DU MODÈLE FÉDÉRAL? CONCLUSION DE LA TABLE RONDE DES EXPERTS, AVEC QUELQUES OBSERVATIONS SUR LA GOUVERNANCE ET LA QUÊTE DU CHANGEMENT *
}

\author{
Mulry Mondélice*
}

\begin{abstract}
Cette contribution présente l'essentiel des réflexions interdisciplinaires présentées sur la question de savoir si l'Union peut faire l'économie du modèle fédéral lors de la table ronde des experts ayant réuni, le 28 avril 2017 à l'Université Laval, des panélistes de différents horizons lors du colloque international sur l'Union européenne - 60 ans du traité de Rome: "tous les chemins mènent-ils encore à Bruxelles?". Un consensus s'est dégagé sur l'idée que l'Union ne peut pas faire l'économie du modèle fédéral. En témoignent des symptômes du fédéralisme : normes - même implicitement -, institutions, agences et politiques, faisant de l'UE une entité se rapprochant d'un État fédéral, avec une répartition des compétences entre l'Union et ses États membres. C'est le cas, par exemple, avec la place reconnue aux droits de la personne qu'illustrent, entre autres, l'existence de l'Agence des droits fondamentaux de l'Union européenne, mais également, l'action humanitaire avec l'Office d'aide humanitaire de l'Union, et la citoyenneté européenne et la mobilité des personnes, ce dernier domaine influençant dans une certaine mesure le Canada. Toutefois, ces symptômes du fédéralisme ne doivent pas faire oublier des pratiques, normes et politiques de l'Union, illustrant un certain fédéralisme en construction. En effet, les Etats membres ne se montrent pas tout à fait prêts à laisser trop de marge de manœuvre à l'Union dans certaines de leurs compétences, notamment en matière d'affaires étrangères, ou dans des domaines sensibles comme l'immigration. Dès lors, cette situation plaçant l'Union dans un entre-deux n'est pas sans conséquences sur le poids de l'Union comme un acteur international. À une époque où le monde fait face à des crises complexes invitant à une gouvernance qui rassemble et mettant à l'épreuve les acteurs internationaux, il serait souhaitable que l'Union montre plus de leadership dans la quête du changement. Dans cette quête, le modèle de gouvernance de l'Union, entre les avancées et défis présents et à venir dans un monde complexe, demeure un enjeu majeur.
\end{abstract}

This article compiles the interdisciplinary contributions on the question of whether the European Union (EU) can dismiss the federal model, presented during the experts round-table discussion reuniting panelists from different horizons on April 28th, 2017, at Laval University, for an international conference on the EU, titled "The Treaty of Rome at 60 Years: Do All Roads Still Lead to Brussels?" A consensus emerged on the idea that the EU cannot dismiss the federal model. Evidence of this stems from symptoms of federalism that can be discerned: norms_ _ even implicitly_, institutions, agencies and policies, rendering the EU an entity resembling a federal state, with a distribution of competences between the Union and its member states. This is the case, for example, with the role attributed to human rights illustrated, among others, by the existence of the European Union Agency for Fundamental Rights, but also with the humanitarian action of the EU through the European Commission's Humanitarian Aid and Civil Protection department, and with European citizenship and mobility rights (which, to a certain extent, influence Canada). However, these symptoms of federalism must not result in forgetting the practices, norms and policies of the EU that demonstrate a federalism under construction. Indeed, member states are not fully ready to allow complete leeway to the EU in all fields of competence, namely as relates to foreign affairs, or to sensitive fields such as immigration. Therefore, this situation places the EU in an in-between phase that is consequential for the relative influence of the Union as an international actor. At a moment during which the world faces complex crises inviting a governance that unites and that challenges international actors, it is advisable that the EU demonstrates more leadership in the context of a quest for change. Within this quest, the

* Cet article, mis à jour, est une version consolidée du rapport final présenté lors des travaux de la table ronde des experts intitulée L'Union européenne peut-elle faire l'économie du modèle fédéral ?", à l'occasion du colloque international L'Union européenne - 60 ans du traité de Rome: Tous les chemins mènent-ils encore à Bruxelles? tenue à l'Université Laval les 27 et 28 avril 2017.

* Chargé d'enseignement en droit international et diplomatie au Collège universitaire Glendon de l'Université York. 
governance model adopted by the EU, between the current and future benefits and challenges in a complex world, remains a significant issue.

Esta contribución presenta lo esencial de las reflexiones interdisciplinarias presentadas sobre la cuestión de saber si la Unión puede prescindir del modelo federal, durante la mesa redonda de los expertos el 28 de abril de 2017 en la Universidad Laval, reuniendo panelistas de diferentes horizontes en el simposio internacional sobre la Unión Europea: 60 años del Tratado de Roma: “¿Todos los caminos siguen conduciendo a Bruselas?" Hubo consenso en que la Unión no puede prescindir del modelo federal. Esto se evidencia en los síntomas del federalismo: estándares, incluso implícitamente, instituciones, agencias y políticas, que hacen de la UE una entidad más cercana a un estado federal, con una división de competencias entre la Unión y sus Estados miembros. Este es el caso, por ejemplo, con el lugar reconocido de los derechos humanos, que se ilustra mediante, entre otras cosas, la existencia de la Agencia de los Derechos Fundamentales de la Unión Europea, pero también la acción humanitaria con La Oficina de Ayuda Humanitaria de la UE, y la ciudadanía europea y la movilidad de las personas (esta última área influye en cierta medida en Canadá). Sin embargo, estos síntomas del federalismo no deben eclipsar las prácticas, normas y políticas de la Unión, lo que ilustra un cierto federalismo en la construcción. En efecto, los Estados miembros no están dispuestos a permitir que la Unión tenga demasiado margen de maniobra en algunos de sus poderes, especialmente en asuntos exteriores, o en áreas sensibles como la inmigración. Por lo tanto, esta situación que coloca a la Unión en un punto intermedio no deja de tener consecuencias sobre el peso de la Unión como jugador internacional. En un momento en que el mundo se enfrenta a crisis complejas que exigen una gobernanza que reúna y ponga a prueba a actores internacionales, sería deseable que la Unión muestre más liderazgo en la búsqueda del cambio. En esta búsqueda, el modelo de gobernanza de la Unión, entre los avances y desafíos presentes y futuros en un mundo complejo, sigue siendo un desafío importante. 
Lorsque, dans le cadre des activités marquant les 60 ans du Traité de Rome ${ }^{1}$, Olivier Delas m'a aimablement demandé de faire la conclusion générale de la table ronde «L'Europe peut-elle faire l'économie du modèle fédéral? », j'ai tout de suite pensé au professeur Peter Leuprecht que j'ai eu l'honneur de remplacer. Souvent, celui-ci fait des conclusions remarquables de nos travaux lors d'activités scientifiques de la Chaire Jean Monnet en intégration européenne de l'Université Laval. Ceux qui ont l'habitude d'écouter P. Leuprecht savent qu'imiter ce grand maître est un exercice périlleux, considérant les défis d'une telle aventure. Toutefois, je m'inspire surtout de sa méthode qui consiste notamment à ne pas citer les noms des intervenants, mais plutôt à évoquer l'essentiel de ce qui a été ou n'a pas été dit. C'est à ce jeu que je me prête ici, en concentrant mes propos sur l'influence de la gouvernance de l'Union européenne (ci-après UE ou Union) sur le poids de celle-ci comme acteur international, alors que des crises politiques et humanitaires, dans leurs liens avec la migration, sont au cœur de son action extérieure.

Au demeurant, il importe de saluer la tenue de cette table ronde sur l'UE et le fédéralisme. Lors de la conclusion d'une notre table ronde sur les 20 ans de l'UE à l'Université Laval en 2012, il a été souligné que «[d]ans cet atelier consacré à l'Europe, il n'a pas été beaucoup question de fédéralisme; comme si en parler était vieux jeu ${ }^{2}$ ». Il est donc heureux de voir que nous nous sommes prêtés au jeu au cours de nos travaux sur les 60 ans des traités de Rome.

Ce jeu a été remarquablement lancé avec l'idée que le fédéralisme apparaît pour l'Union européenne comme une question «existentielle, c'est-à-dire, être ou ne pas être une entité fédérale », le sujet étant vieux comme l'UE elle-même. Il a été rappelé qu'au fondement même de l'UE, Victor Hugo avait déjà parlé des «ÉtatsUnis d'Europe ${ }^{3}$ », alors que la mention d'une "fédération» dans la Déclaration Schuman du 9 mai $1950^{4}$, illustre «un gène fédéral ». En pratique, ce fédéralisme s'est apparenté à quelque chose à construire au fur et à mesure. En effet, ce terme n'est pas mentionné dans les traités, car "fédéralisme, constitution et loi sont comme des interdits», mais tout comme existe une sorte de «grammaire européenne », il existe une « grammaire du fédéralisme de l’UE », « objet juridique non identifié ».

Cette remarquable mise en contexte fait écho à la répartition des compétences entre l'UE et ses États membres, ainsi qu'il ressort des articles 2 à 6 du Traité sur le fonctionnement de l'Union européenne ${ }^{5}$, version consolidée issue du Traité de Lisbonne ${ }^{6}$. La table ronde a ainsi permis des débats intéressants sur deux

1 Traité de Rome instituant la communauté économique européenne, 25 mars 1957, 298 RTNU 11 (entrée en vigueur : $1^{\mathrm{er}}$ janvier 1958) [TFUE].

2 Peter Leuprecht, «Conclusion de notre table ronde des experts: quel avenir pour l'Union européenne », en ligne: (2012) RQDI numéro spécial à la p 197 <www.sqdi.org/wpcontent/uploads/HS-UE_15_Leuprecht.pdf $>$.

3 Victor Hugo, discours d'ouverture, Congrès international de la paix, présenté à Paris, 21 août 1849.

4 Fondation Robert Schuman, «Déclaration du 9 mai 1950 prononcée par Robert Schuman », Question d'Europe, 2011, en ligne : <www.robert-schuman.eu/fr/doc/questions-d-europe/qe-204-fr.pdf>.

5 TFUE, supra note 1.

6 Traité de Lisbonne modifiant le traité sur l'Union européenne et le traité instituant la Communauté européenne, 13 décembre 2007, RT CIG 14/07 (entrée en vigueur : 1er décembre 2009). 
points principaux. D'une part, le fédéralisme de l'UE fait consensus. La réponse à la question de la table ronde semble claire : l'UE ne peut pas faire l'économie du modèle fédéral, fédéralisme réel qu'illustre également la matière humanitaire (I). D'autre part, ce fédéralisme demeure un fédéralisme à la pièce, pas tout à fait complet et qui reste en construction (II). Ce modèle de gouvernance invite dès lors à s'interroger sur la réputation et la puissance de l'UE comme acteur international dans un monde en quête de changement face à des crises complexes (III).

\section{L'UE ne peut pas faire l'économie du modèle fédéral}

Dans la nature même de l'UE, se trouvent quelques «symptômes du fédéralisme », sorte d'éléments caractéristiques de l'État fédéral. Une illustration particulière de ce fédéralisme est le traitement accordé à la personne dans l’Union.

\section{A. Des symptômes du modèle fédéral dans la nature même de l'Union}

En 1957, les États membres fondateurs ont adopté un traité et créé une entité non souveraine, soumise au principe de spécialité, caractéristique d'une organisation internationale au regard du droit international public. Mais ce sujet dérivé est plus qu'une organisation internationale, d'autant plus que dans le système des traités, l'Union offre des «garanties de droits fondamentaux ». En effet, bien qu'au double niveau universel et régional, des organisations internationales promeuvent les droits de la personne, les garanties de droits fondamentaux renvoient plus à la terminologie $\mathrm{du}$ droit constitutionnel. Sur le plan matériel, outre les droits de la personne, avec la répartition et le transfert des compétences, une méthode intergouvernementale faisant place peu à peu à une méthode communautaire au sein de cette «organisation internationale », on est donc dans un modèle «fédéral ». Le modèle de gouvernance de l'État canadien peut servir à illustrer ce modèle fédéral, avec les articles 91 et 92 de la Loi constitutionnelle de $1867^{7}$, et plus généralement la Constitution du Canada incluant la Charte canadienne des droits et libertés de $1982^{8}$. D'une certaine manière, le fédéralisme est pour l'essentiel caractérisé par une répartition des compétences entre les deux paliers fédéral et provincial de gouvernement et une importante place accordée aux droits de la personne. Ces éléments se trouvent au cœur de l'Union européenne et du Canada, deux partenaires dans un lien privilégié en raison de valeurs et priorités communes.

Au sein de l'UE, sont constatés des « symptômes » ou « données fédérales », avec des politiques et organes de sources variées, notamment dans des traités et des mécanismes diplomatiques. Parmi ces symptômes figurent: la subsidiarité, la consécration de la théorie de l'administration indirecte ou déléguée, l'union

Loi constitutionnelle de 1867 (R-U), 30 \& 31 Vict, c 3, art 91-92 reproduite dans LRC 1985, annexe II, $\mathrm{n}^{\circ} 5$.

8 Charte canadienne des droits et libertés, partie I de la Loi constitutionnelle de 1982, constituant l'annexe B de la Loi de 1982 sur le Canada (R-U), 1982, c 11. 
économique et monétaire, des agences- dont l'Agence des droits fondamentaux de l'Union européenne- et le mandat d'arrêt européen.

En matière diplomatique, le Service européen pour l'action extérieure (SEAE), dirigé par un Haut représentant pour les affaires étrangères et la politique de sécurité, se veut une sorte de ministère des Affaires étrangères de l'UE. Celle-ci est ainsi représentée par près de 140 délégations auprès d'États non membres de l'UE et d'organisations internationales à travers le monde. Les États membres gardent toutefois leurs propres relations diplomatiques avec les États et organisations internationales, bien que le personnel du SEAE inclue des membres du service diplomatique de ses États membres. Cette structure institutionnelle n'est pas sans rappeler une certaine évolution au sein des ministères des Affaires étrangères et de la pratique des États impliquant de plus en plus des acteurs infra-étatiques dans les affaires étrangères.

Par ailleurs, la matière financière, avec le Système européen de banques centrales, la Banque centrale européenne, le comité économique et financier et l'Eurogroupe, peut illustrer une volonté d'accorder plus de poids au communautaire. Plus particulièrement, l'union douanière et la politique commerciale sont une expression manifeste du fédéralisme au sein de l'UE.

Ce n'est pas étonnant que l'économie soit le domaine prioritaire, avec une place centrale dans le Traité de Rome de 19579, dans le contexte d'une Europe ravagée par la guerre. Au fil des années, il est notable de voir que la circulation des personnes constitue un élément important de la logique fédérale dont bénéficient les citoyens européens.

Il peut également être noté qu'en matière d'aide humanitaire, les rôles de la Direction générale pour la protection civile et les opérations d'aide humanitaire de la Commission européenne (ECHO), organe chargée de la protection civile et des opérations d'aide humanitaire européennes, attestent du fédéralisme dans la promotion des valeurs humanistes par l'UE à l'échelle mondiale. Si l'action humanitaire de l'UE est confrontée à un manque de coordination entre l'Union et ses États membres, elle n'en est pas moins un «symptôme» du fédéralisme. Elle s'inscrit dans l'action extérieure de l'UE et gagne de plus en plus en visibilité par rapport aux actions d'aide humanitaire menées par ses États membres isolément dans ce domaine sensible, au cœur de la promotion de la sécurité humaine dans les relations internationales ${ }^{10}$.

Au cours des vingt-cinq dernières années, l'UE s'est posée comme un acteur humanitaire de poids à l'échelle mondiale, que ce soit dans des situations marquées par des catastrophes naturelles, comme le séisme dévastateur du 12 janvier 2010 en Haïti, ou des conflits, et ce, des Balkans et en Afrique vers les années 1990 au Moyen-Orient aujourd'hui avec la guerre en Syrie. L'ampleur de cette dernière crise

9 TFUE, supra note 1.

10 Michael Schloms, «Le dilemme inévitable de l'action humanitaire» (2005) 60 Cult \& Confl aux pp 85-102. 
et ses conséquences sur l'Europe ont conduit à une évolution de l'humanitaire au sein de l'Europe elle-même, notamment avec les «fonds d'aide» accordés par l'UE à la Grèce pour la gestion des réfugiés. Pour la première fois, cette crise a conduit à un élargissement de l'aide humanitaire de l'UE: d'une action pensée pour la solidarité envers le reste du monde, l'UE est passée à une action humanitaire au sein de l'Union elle-même. C'est ce que traduit l'adoption, le 16 mars 2016, du Règlement (UE) 2016/369 du Conseil du 15 mars 2016 relatif à la fourniture d'une aide d'urgence au sein de l'Union ${ }^{l l}$.

L'Union illustre dès lors un « fédéralisme juridique, intérieur, coopératif, une fédération d'États-nations ». Bien que sur le plan politique, du chemin reste à parcourir pour une diplomatie plus agissante, ces limites n'évacuent pas la nature fédérale de l'UE, également illustrée par la place accordée par celle-ci à la personne.

\section{B. Un fédéralisme illustré par la place accordée à la personne au sein de l'Union}

Le fédéralisme a une dimension libérale reposant sur le traitement accordé à la personne, qu'elle soit physique ou morale. Ici encore, comme des modèles transitoires dans certains pays, l'UE apparaît comme un fédéralisme en évolution, que ce soit au titre de la nationalité européenne ou de la place accordée aux droits fondamentaux.

D'un côté, « la nationalité dans la fédération européenne » montre que l'UE n'est pas - encore - un État, mais on n'en est pas loin non plus. Avec la citoyenneté européenne, la personne jouit d'une série de droits à l'intérieur de l'espace européen. C'est là l'idée du fédéralisme évolutif, puisque cette nationalité européenne s'est construite et se construira encore par étapes, dans le temps et selon le degré d'intégration voulu par les États membres. Reste qu'il faudrait également prendre en compte l'opinion des populations au sein desdits États pour rendre compte du degré d'adhésion au projet européen.

À cet égard, l'aide humanitaire de l'UE - qui place celle-ci parmi les premiers donateurs à l'échelle mondiale et s'inscrivant dans sa diplomatie ${ }^{12}$ - semble trouver une certaine adhésion de la population au nouveau corps des Volontaires de l'aide humanitaire de l'UE, prévu à l'article 214 (5) du TFUE. Il est intéressant de noter que le financement de cette initiative, comme du programme « [1]'Europe pour les citoyens pour la période 2014-202013», est assuré par l'Agence communautaire pour l'Éducation, l'audiovisuel et la culture (EACEA), agissant par ailleurs dans les domaines de la citoyenneté, de l'éducation, de la culture, de l'audiovisuel et du sport.

11 Règlement (UE) 2016/369 du Conseil du 15 mars 2016 relatif à la fourniture d'une aide d'urgence au sein de l'Union, JOUE,16.3.2016, L 70/1.

12 Joanna Dobrowolska-Polak, "Humanitarian Diplomacy of the European Union» (2014) 5 New Diplomacy in Open Eur aux pp 118-119.

$13 \mathrm{CE}$, Commission, Guide du programme concernant les subventions pour actions : Programme «l'Europe pour les citoyens » 2014-2020, Bruxelles, 2018. 
L'importance d'une telle agence est notable. En effet, dans l'intégration européenne, la culture et le dialogue entre les populations demeurent essentiels pour le citoyen appelé à mieux intérioriser les valeurs de l'Union et l'importance de celle-ci dans une gouvernance cherchant des solutions communes face à ses divers États membres.

De l'autre côté, le fédéralisme européen se traduit par la protection des droits fondamentaux des individus contre les communautés et, avec la Charte des droits fondamentaux de l'UE ${ }^{14}$, contre les États lorsqu'ils mettent en œuvre le droit de l'Union. Considérant le développement du système de protection des droits fondamentaux au sein de l'UE, un parallèle a été envisagé avec l'adoption de la Charte des droits fondamentaux de l'UE et de la Charte canadienne des droits et libertés ${ }^{15}$, celle-ci protégeant l'individu contre l'action du gouvernement tant fédéral que provincial.

Il apparaît curieux de voir à quel point un des «symptômes » du fédéralisme européen, à savoir l'Agence des droits fondamentaux de l'Union européenne (FRA), constitue un élément important de ce système. En effet, créé en 2007, cet organe illustre la réception, à l'échelle d'une organisation internationale, du concept d'institution nationale des droits de l'homme (INDH), entendue comme une institution créée par l'État dans le but de promouvoir et de protéger les droits et libertés. Au nombre de 120 aujourd'hui, les INDH voient leur nature et leur fonctionnement régis par les Principes de Paris relatifs au fonctionnement des Institutions nationales des droits de l'homme, normes universelles avec lesquelles les États doivent se conformer s'ils souhaitent obtenir l'accréditation du sous-comité d'accréditation lorsqu'ils créent une $\mathrm{INDH}^{16}$.

La FRA se veut un agent catalyseur de changement en douceur, « velvet Globe $»{ }^{17}$, formule employée pour rendre compte du rôle majeur que peuvent jouer les INDH, avec lesquelles travaille l'AFR, dans la gouvernance notamment au niveau européen. Pour autant, nous savons que les principales conditions de succès de telles institutions demeurent, entre autres, le soutien politique et le courage et la détermination de leur responsable, la qualité de la coopération avec les pouvoirs législatif, exécutif et judiciaire. Considérant le rôle crucial que peuvent jouer les institutions nationales dans l'amélioration effective des droits et libertés, il serait souhaitable que l'UE associe plus avant la FRA et les institutions nationales des droits de l'homme dans les relations entre l'Union européenne et les États tiers. Une telle démarche pourrait être envisagée notamment dans la coopération au développement et l'aide humanitaire, par des mécanismes de coopération bilatérale ou

14 Charte des droits fondamentaux de l'Union européenne, constituant l'art 6 du Traité sur l'Union européenne, 2000, c 364/01.

15 Charte canadienne des droits et libertés, supra note 9.

16 Emmanuel Decaux, «Le dixième anniversaire des Principes directeurs des Institutions nationales des droits de l'homme dits "Principes de Paris"» (2003) 3 Dr fondamentaux aux pp 13 \& 28. Voir aussi Jan Wouters et Katrien Meuwissen, dir, National human rights institutions in Europe : comparative, European and international perspectives, Cambridge, Intersentia Publishing, 2013.

17 Shelagh Day, Lucie Lamarche et Ken Norman, 14 Arguments in favour of National Human Rights Institutions, Toronto, Irwin Law, 2014 à la p 4. 
multilatérale. En effet, il semble important de mieux s'approprier et d'étendre le rôle de telles institutions notamment compte tenu de l'analyse qu'elles pourraient fournir à propos des impacts de projets financés par l'UE sur les droits fondamentaux, et ce, en amont et en aval. Si la FRA joue déjà un rôle dans la diplomatie des droits de la personne, notamment avec ses études, rapports et échanges de pratiques avec les INDH européennes ${ }^{18}$, ses potentialités ne sont toutefois pas encore suffisamment explorées, incluant dans la protection juridictionnelle des droits.

Il est vrai qu'une telle approche suscite de la prudence en vertu de l'approche diplomatique caractérisant le travail de la FRA comme toute institution de cette nature au sein d'une organisation internationale. Toutefois, à l'instar du Haut-Commissaire aux droits de l'homme du Conseil de l'Europe, on pourrait se demander si l'évolution de la nature fédérale de l'Union ne devrait pas conduire à la possibilité pour la FRA de saisir la Cour de justice de l'UE en cas de violations massives des droits, ou encore d'agir devant celle-ci à titre de tierce intervenante dans le cadre d'affaires présentant un enjeu majeur pour la protection des droits au sein de l'UE. Une telle proposition n'apparaît pas étrange à l'observateur de l'action de la Commission canadienne des droits de la personne pouvant, en droit canadien, saisir le Tribunal canadien des droits de la personne ou agir devant la Cour suprême à titre d'intervenante.

Si les caractéristiques du modèle fédéral que l'on retrouve dans la nature de l'UE ont ainsi fait l'objet d'un consensus autour de la table, les intervenants ont toutefois observé qu'il s'agit d'un «fédéralisme à la pièce» et de surcroit, en construction.

\section{L'UE révèle un fédéralisme toujours en construction}

Le fédéralisme de l'UE est souvent confronté à un manque de volonté politique chez les responsables des États membres, ce qui renvoie la balle dans le camp de ces derniers dans une série de domaines, notamment l'immigration, le budget et la politique étrangère. C'est dire que les États ne sont pas vraiment prêts à transférer à l'Union certaines compétences nationales. Il a été rappelé que dans le domaine des droits de la personne et de l'état de droit par exemple, l'affaire Aurore Martin, française remise en 2012 à la justice espagnole suite à un mandat d'arrêt européen- procédure d'entraide judiciaire pénale remplaçant l'extradition classique- et libérée en 2016, révèle cette tension entre intégration par la méthode communautaire et la volonté des États ${ }^{19}$. Pourtant, la Cour de justice a développé le principe de «confiance mutuelle», au cœur de la reconnaissance mutuelle, élément clé de l'espace de liberté, de sécurité et de justice devant guider les relations entre l'UE et ses États membres.

18 Sur l'UE et la diplomatie des droits de la personne voir Toby King, « The European Union as a Human Rights Actor» dans Michael O'Flaherty et al, dir, Human Rights Diplomacy: Contemporary Perspectives, Boston, Martinus Nijhoff Publishers, 2011 aux pp 77-100.

19 Affaire Aurore Martin (France c Aurore Martin), Mandat d'arrêt européen contre Aurore Martin (23 novembre 2010), Cour d'appel de Pau (sér C) n 389/2010. 
Le Brexit a été regardé comme une expression de la faiblesse du fédéralisme européen, incompatible avec l'idée même du fédéralisme au niveau national. Dans le sillage du Brexit, il a été souligné que dans le discours politique, s'est répandue l'idée que le fédéralisme reste une menace pour l'UE.

Dans le domaine de l'économie, au sein de l'UE, définir un système fiscal par exemple constitue un défi. Il a été signalé d'ailleurs qu'il importe de considérer ces faiblesses du fédéralisme européen à l'échelle internationale. En particulier, il a été souligné qu'en matière de négociation et de ratification de traités, l'UE peut se révéler un partenaire peu fiable pour ses partenaires étrangers en raison de la procédure de conclusion des traités sous l'empire du Traité de Lisbonne (art. 218 TFUE), ainsi que l'ont montré les interactions entre l'UE, la Belgique et le parlement de Wallonie dans le cadre de la procédure de ratification de l'Accord économique et commercial global $^{20}$. Dès lors, l'enjeu est de réconcilier politique économique et volonté politique. En effet, si la politique commerciale reste une compétence exclusive de l'UE, la mondialisation et la réglementation croissante qu'elle nourrit, font tomber des traités dans la catégorie des accords mixtes faisant intervenir les États. De tels accords peuvent être regardés comme une expression de la faiblesse du fédéralisme de l'UE.

À propos, il est à remarquer que tous les partenaires de l'UE ne lui font pas les mêmes exigences. Par exemple, dans les relations entre l'UE et le groupe d'États Afrique, Caraïbe, Pacifique, les accords de partenariat économique $(A P E)^{21}$ conclus n'ont pas toujours obtenu la confiance éclairée des populations concernées ${ }^{22}$. En particulier, l'APE conclu en 2008 avec les États du sous-groupe caraïbe (CARIFORUM), a été accepté sans trop grande information du public et sans débat sur les questions environnementales ou sur le mécanisme du règlement des différends entre investisseurs et États, à l'exception d'Haïti qui ne l'a pas ratifié. Avec la technique de l'entrée en vigueur provisoire, l'Accord CARIFORUM-UE, le premier $A P E$ pouvant être rangé dans la catégorie des accords de nouvelle génération, a pu contribuer à faciliter une certaine mobilité pour certains professionnels d'États caribéens au sein de l'espace européen, sous réserve des subtilités liées aux visas spécifiques pour les régions ultras périphériques françaises de l'UE (notamment Guyane, Martinique, Guadeloupe).

C'est dire que les relations économiques de l'UE tendent à influencer la pratique vers une certaine mobilité des professionnels. Il a été noté à propos que dans ce domaine, le fédéralisme de l'UE apparaît, d'une certaine manière, plus poussé que celui du Canada, ce qu'illustre la conclusion le 7 avril et l'entrée en vigueur le 1er

20 TFUE, supra note 1, art 218. Accord économique et global, Canada et 1'Union européenne, octobre 2016, RT can 460 (entrée en vigueur : 21 septembre 2017) [AECG].

21 Commission européenne, "Les pays d'Afrique, des Caraïbes et du Pacifique (ACP) », en ligne : $<$ ec.europa.eu/taxation_customs/business/calculation-customs-duties/rules-origin/general-aspectspreferential-origin/arrangements-list/countries-africa-caribbean-pacific-acp_fr>.

22 Claire Guillemin, «L'Accord de partenariat économique Union européenne-CARIFORUM : une réponse à l'évolution des relations UE-ACP dans le contexte international », dans Dorval Brunelle dir, Repenser l'Atlantique : Commerce, immigration, sécurité, Bruxelles, Bruylant, 2012 aux pp 69 à 76. 
juillet 2017 de l'Accord de libre-échange canadien (ALÉC) entre les provinces canadiennes dans le sillage de l'Accord économique et commercial global entre l'UECanada. À la suite de la conclusion de l'AECG, l'ALÉC est apparu nécessaire pour le système fédéral canadien non contesté, mais apparu quelque peu "coincé » pour les entreprises provinciales au regard de l'ouverture de marchés dans d'autres provinces aux compagnies européennes. Ce serait là une influence du fédéralisme de l'UE, voire des relations transatlantiques, sur les relations économiques à l'intérieur du Canada. S'il reste évident que l'UE est appelée à faire évoluer les relations économiques, il est également urgent pour celle-ci de se poser en acteur de changement cohérent dans un monde confronté à diverses crises.

\section{Le fédéralisme pose des défis pour une action extérieure efficace de l'Union}

Les réticences soulignées précédemment traduisent la difficulté pour l'UE de trouver une voie claire entre l'interdépendance qui caractérise les liens entre les membres de la société internationale et des pratiques peu cohérentes de ces derniers dans la quête du changement. Il en est ainsi en matière d'immigration et de crise humanitaire, où se confrontent, d'un côté, les valeurs promues par l'UE, et, de l'autre, la souveraineté des États membres, manifestée dans le sens opposé des droits lorsque sont en jeu notamment des préoccupations économiques et politiques. L'exemple de la Déclaration UE-Turquie du 18 mars 2016 reste très parlant sur ce point ${ }^{23}$.

L'actualité de la pensée des fondateurs de l'UE a été soulignée avec force. En effet, Jean Monnet a dit: «nous n'unissons pas les États, nous unissons les hommes ». Or, de nos jours, au sein de l'UE, bien qu'il y ait eu quelque évolution sur le plan institutionnel et juridique allant dans le sens de la promotion de la dignité humaine, la question migratoire a dévoilé les contradictions au cœur de l'Union. En effet, avec la situation syrienne en particulier, nous constatons qu' «Union de valeurs ", l'UE a du mal à promouvoir et protéger ces dernières en temps de crise, surtout envers les plus vulnérables. La question est donc de savoir comment l'UE pourrait mener une politique migratoire cohérente avec ses valeurs. L'enjeu, c'est de réfléchir sur la complexité des voies du changement par des mesures propres à concilier les intérêts souvent divergents de l'Union, de ses États membres, des États tiers et ceux des personnes se trouvant sous leur juridiction. On a souligné avec raison la manière dont le manque de solidarité entre États membres entrave la prise de décisions courageuses au sein l'Union. Cette remarque sous-tend également la sélectivité pratiquée par l'UE et ses États membres dans la promotion des droits, incluant dans ses relations avec les États tiers. Il me semble que cette solidarité, que ce soit dans le cadre de l'aide humanitaire ou la coopération au développement, pose quelques difficultés quant au message de l'UE auprès desdits États et de leur population.

23 Déclaration entre l'Union européenne et la Turquie, Conseil, Communiqué de presse 144/16 du 18 mars 2016, en ligne < https://www.consilium.europa.eu/fr/press/press-releases/2016/03/18/eu-turkeystatement/pdf $>$ 
Comme il a été souligné précédemment, dans le contexte de l'Union, le fédéralisme fait face à la volonté des États membres. On a notamment parlé de migration, de sécurité, de la lutte contre le terrorisme, auxquels on peut ajouter l'aide humanitaire. L'exemple du système Dublin, tombé sous la méthode communautaire au détriment de la méthode intergouvernementale, mais remis en cause dans le contexte de la gestion des mouvements migratoires en lien avec la situation syrienne, montre les difficultés d'une réalisation effective du fédéralisme au sein de l'UE et fragilise celle-ci comme acteur international.

Le concept de différenciation dont il a été question dans les échanges peut fournir une bonne grille d'analyse, du point de vue de la justification de la réticence affichée par certains États dans certains domaines. Un ouvrage résultant des actes d'un colloque très intéressant tenu à McGill sur Human Rights and diverse societies fourmille de leçons les défis que pose la gouvernance dans la diversité au plan interne et dans les relations internationales ${ }^{24}$. Il s'en est dégagé un consensus sur l'idée selon laquelle, au-delà des normes juridiques, la complexité de nos sociétés à l'époque contemporaine (marquée notamment par les enjeux électoraux, la crise économique, contact entre le global et le local, l'immigration...) met les États dans un réseau d'interdépendance pourtant malmenée par la sélectivité pratiquée par les États.

Nous avons enfin beaucoup parlé de la place de l'économie, «noyau dur de la politique étrangère $»^{25}$ de l'Union et sa prise en compte dans la quête de changement. Pour autant, comme le montrent les relations Nord-Sud, notamment les États ACP, la place réservée à l'économie de marché se double de la nécessité de tenir compte des circonstances particulières de l'économie et des inégalités dans les sociétés concernées. Or, souvent, on assiste à une promotion du libre-échange qui favorise les intérêts de multinationales au détriment de l'amélioration des conditions de vie des populations. Il en est de même des politiques misant sur la sécurité et la répression au nom de la lutte contre l'immigration irrégulière ou de la criminalité transnationale, avec des réformes pénales soutenues par la coopération internationale impliquant l'Union. Se crée ainsi une certaine sélectivité entre les droits économiques, sociaux et culturels et les droits civils et politiques, oubliant l'indivisibilité et l'interdépendance des droits. Cette sélectivité rime avec la différenciation, de sorte que dans le contexte européen, les intérêts particuliers des États sont souvent mis de l'avant au détriment d'une vision commune qui serait mise en œuvre par des institutions communautaires. Pour autant, il semble que les crises, comme il a été souligné, font avancer vers plus d'Europe. On a ainsi posé la question de savoir s'il ne faudrait pas plus d'Europe, question restée ouverte.

C'est autant dire que nombreux sont les défis que pose la nature fédérale de l'Union pour une action efficace de celle-ci face à la situation inquiétante à l'échelle mondiale. En effet, la société internationale est confrontée à une incertitude marquée

24 François Crépeau et Colleen Sheppard, Human Rights and Diverse Societies: Challenges and possibilities, Cambridge, Cambridge Scholars Publishing, 2013.

25 Franck Petiteville, «La coopération économique de l’Union européenne entre globalisation et politisation » (2001) $51: 3$ Rev fr de Science P aux pp 431-458. 
en particulier par des relations difficiles avec l'administration américaine actuelle, des discours qui nourrissent le populisme, le chômage et le sous-emploi et des inégalités sociales criantes, l'endettement, des guerres et des catastrophes naturelles mettant la sécurité humaine en danger. Un tel contexte rend certes difficile l'attribution par les États membres, de compétences plus poussées à l'UE. En même temps, l'UE et ses États membres se retrouvent devant un choix entre l'interdépendance qui rassemble et la différenciation égoïste qui ruine l'humanité. Pourtant, l'enjeu pour la diplomatie de l'UE est de parler d'une seule voix et d'envoyer un message clair dans la recherche de la paix et la sécurité internationales, du développement et des droits de la personne. Dès lors, bien que le réalisme politique et économique ne nourrisse pas une telle perspective, c'est au prix de plus d'information, de consultation, de participation et de solidarité, que la gouvernance européenne pourrait conduire au changement que souhaitent les populations européennes et des États tiers que touche son action.

Il a été rappelé qu' « une Europe fédérale, c'est une Europe capable » et, c'est en avançant qu'elle sera plus capable. Dès lors, comme le disait Aimé Césaire, souhaitons que l'UE fasse «un pas, un autre pas, encore un autre pas et tenir gagné chaque pas », phrase reprise dans la pièce «Tragédie du roi Christophe ${ }^{26} »$.

26 Aimé Césaire, Pièce de théâtre, La tragédie du roi Christophe, Martinique, Présence Africaine, 4 août 1964. 